\title{
Duração do sono, ingestão de energia e macronutrientes em indivíduos com síndrome metabólica
}

\author{
Sleep duration, energy and macronutrient intake in individuals with Metabolic syndrome \\ Naiara Brunelle Oliveira Neiva', Luama Araújo dos Santos², Edilene Maria Queiroz Araújo ${ }^{3 *}$ \\ ${ }^{1}$ Mestranda do Programa de Pós-graduação Processos Interativos dos Órgãos e Sistemas. Instituto de Ciências da \\ Saúde. Universidade Federal da Bahia - UFBA; ${ }^{2}$ Doutoranda do Programa de Pós-graduação Processos Interativos dos \\ Órgãos e Sistemas. Instituto de Ciências da Saúde - UFBA; ${ }^{3}$ Doutora em Biotecnologia e Genética pela Universidade \\ Estadual de Feira de Santana. Professora Adjunto da Universidade do Estado da Bahia - UNEB e do Programa de Pós- \\ graduação Processos Interativos dos Órgãos e Sistemas. Instituto de Ciências da Saúde - UFBA
}

\begin{abstract}
Resumo
Introdução: a duração do sono tem sido associada à síndrome metabólica e o principal mediador entre tais fatores parece ser a dieta, pois a ingestão de quilocalorias e macronutrientes pode variar conforme as horas dormidas. Objetivo: avaliar se existe associação entre duração do sono e o consumo de energia e de macronutrientes em indivíduos com síndrome metabólica. Metodologia: tratase de um estudo retrospectivo, transversal, com dados secundários de prontuários de pacientes adultos, atendidos entre os anos de 2013-2020. A duração do sono foi categorizada em: curta para $\leq 6$ horas por noite; adequada para 7-8 horas por noite; e longa para $\geq 9$ horas por noite; já os dados dietéticos foram obtidos por meio da aplicação e cálculo de recordatórios alimentares de 24 horas. Após ajustes para idade e sexo, as variáveis foram avaliadas por meio do teste Qui-Quadrado, sendo considerados significativos valores de $p<0,05$. Resultados: das 375 pessoas avaliadas, quase metade relatou uma curta duração de sono; entretanto, não foram encontradas associações estatisticamente significativas entre a duração do sono e o consumo calórico ( $p=0,957)$, de carboidratos ( $p=0,975)$, proteínas $(p=0,865)$ e lipídios $(p=0,382)$. Discussão: a maior prevalência encontrada de curta duração do sono pode estar associada a algumas características sociodemográficas da população estudada, como o sexo feminino, o sedentarismo, o aumento da idade, a baixa renda e a ocupação ativa no mercado de trabalho. Conclusão: indivíduos com síndrome metabólica parecem ter menor tempo de sono, contudo, a associação do sono com o consumo alimentar ainda requer maiores investigações.

Palavras-chave: Síndrome metabólica. Sono. Ingestão de energia. Macronutrientes.
\end{abstract}

\begin{abstract}
Introduction: Sleep duration has been associated with metabolic syndrome and the main mediator between these factors seems to be diet, as the ingestion of kilocalories and macronutrients can vary according to the hours of sleep. Objective: to assess whether there is an association between sleep duration and energy and macronutrient intake in individuals with metabolic syndrome. Methods: this is a retrospective, cross-sectional study, with secondary data from medical records of adult patients, treated between the years 20132020. Sleep duration was categorized into: short for $\leq 6$ hours per night; suitable for 7-8 hours per night; and long for $\geq 9$ hours per night; dietary data were obtained through the application and calculation of 24-hour dietary recalls. After adjustments for age and sex, the variables were evaluated using the Chi-Square test, with values $p<0.05$ being considered significant. Results: of the 375 people surveyed, nearly half reported a short sleep duration; however, no statistically significant associations were found between sleep duration and caloric intake $(p=0.957)$, carbohydrates $(p=0.975)$, proteins $(p=0.865)$ and lipids $(p=0.382)$. Discussion: the higher prevalence of short sleep duration found may be associated with some sociodemographic characteristics of the studied population, such as female gender, sedentary lifestyle, increasing age, low income and active occupation in the labor market. Conclusion: Individuals with metabolic syndrome seem to have less sleep time; however, the association of sleep with food consumption still requires further investigation. Keywords: Metabolic Syndrome. Sleep. Energy Intake. Macronutrients
\end{abstract}

\section{INTRODUÇÃO}

A duração do sono é um importante indicador de saúde, especialmente para o equilíbrio cardiometabólico (WHINNERY et al., 2014; ZURAIKAT et al., 2020). Pesquisas observaram que existe uma associação entre curtos períodos de sono e os distúrbios associados à síndrome metabólica (SM), tais como, obesidade (WU; ZHAl;

Correspondente/Corresponding:*Edilene Maria Queiroz Araújo - End.: Rua Embira, no 149, Edifício Paladio, Apto 1204, Greenvile/ Patamares. CEP 41680-113 - Tel: (71) 3561-8818 - E-mail: dilaq@msn.com
ZHANG, 2014), diabetes tipo 2 (CAPPUCCIO et al., 2010a), doenças cardiovasculares (CAPPUCCIO et al., 2011; YIN et al., 2017), além de aumento da mortalidade (CAPPUCCIO et al., 2010b). Do lado oposto, a longa duração do sono também está associada a um aumento do risco de morbidade e mortalidade (CAPPUCCIO et al., 2010a). Assim, nota-se uma curva em $U$ de correlação entre tempo de sono e a SM (JU; CHOI, 2013).

Nesse contexto, tem sido proposto que o maior mediador da relação entre o sono e o risco cardiometabólico seja a dieta (NISHIURA; NOGUCHI; HASHIMOTO, 2010). O 
sono insuficiente ou em excesso pode promover mudanças na ingestão calórica, no perfil de nutrientes (DASHTI et al., 2015; ST-ONGE; MIKIC; PIETROLUNGO, 2016) e nas escolhas alimentares (ALIMENTI et al., 2021; DASHTI et al., 2015; GRANDNER et al., 2013), o que levaria ao ganho de peso, resistência à insulina e outras desordens (REITER et al., 2012). Contudo, os mecanismos envolvidos nesse processo ainda requerem maior investigação (ZURAIKAT et al., 2020) e por isso, a crononutrição tem ganhado destaque como um campo de pesquisa sobre o sono e os comportamentos alimentares durante a vigília (CASTRO et al., 2019).

O balanço energético tem um papel importante no desenvolvimento da SM que, por sua vez, é influenciado pelo perfil de macronutrientes ingeridos. Dietas com maior teor de carboidratos e lipídios geralmente têm maior densidade energética, contribuindo para um superavit calórico (NISHIURA; NOGUCHI; HASIMOTO, 2010). Portanto, compreender a relação entre o sono e o consumo alimentar pode abrir caminhos para novas estratégias de prevenção e tratamento da SM, por serem fatores de risco modificáveis pelo estilo de vida (SANTOS; FERRARI; FERRARI, 2015).

Assim, o objetivo deste estudo foi avaliar se existe associação entre duração do sono e o consumo de calorias totais diárias e de macronutrientes (carboidratos, proteínas e lipídios) em indivíduos com SM.

\section{METODOLOGIA}

\section{a. Aspectos éticos}

Este projeto de pesquisa foi aprovado pelo Comitê de Ética em Pesquisa da Universidade do Estado da Bahia (CAAE: 03409712.9.3001.5023).

\section{b. Tipo e delineamento do estudo}

Trata-se de um estudo retrospectivo, transversal, com dados secundários de prontuários de pacientes atendidos entre os anos de 2013-2020. A população foi composta por pacientes adultos, de ambos os sexos, com SM, encaminhados do Hospital Geral Roberto Santos ou por demanda espontânea e atendidos no Núcleo de Pesquisa e Extensão em Genômica Nutricional e Disfunções Metabólicas (GENUT), da Universidade do Estado da Bahia.

\section{c. Coleta de dados}

Todos os dados foram coletados por membros da equipe do GENUT, incluindo estagiários e nutricionistas devidamente treinados, que realizaram as seguintes etapas: explicação acerca dos objetivos da pesquisa e coleta da assinatura do Termo de Consentimento Livre e Esclarecido (TCLE); aplicação de uma ficha de anamnese nutricional, composta de inquérito sociodemográfico; inquérito alimentar; história clínica e familiar; avaliação antropométrica, com aferição de peso e altura e circunferência da cintura; aferição da pressão arterial sistêmica, por meio de estetoscópio e tensiômetro, com métodos conforme I Diretriz brasileira de diagnóstico e tratamento da síndrome metabólica (CARVALHO, 2005); e, por fim, coleta sanguínea para a realização das dosagens bioquímicas de HDL-c, triglicerídeos e glicemia de jejum, pelo método de colorimetria enzimática. $O$ diagnóstico de SM foi definido segundo os critérios propostos pela Federação Internacional de Diabetes (ALBERTI; ZIMMET; SHAW, 2006).

\section{d. Duração do sono}

Para investigar a duração do sono, os avaliadores fizeram a seguinte pergunta: "Quantas horas você dorme por noite, em média?". Após a seleção dos prontuários, foram categorizados três grupos: curta duração para tempo de sono $\leq 6$ horas por noite; adequada duração para 7-8 horas por noite; e longa duração para tempo de sono $\geq 9$ horas por noite, conforme estabelecido por Kant e Graubard (2014) e Castro et al. (2019).

\section{e. Dados dietéticos}

Os dados sobre a dieta foram colhidos por meio da aplicação de um recordatório alimentar de 24 horas, conforme utilizado por Grandner et al. (2013) e Castro et al. (2019). Os recordatórios forneceram a descrição de todos os alimentos habitualmente consumidos pelos participantes em dias típicos, suas respectivas quantidades e formas de preparo, além do horário das refeições. Descartaram-se relatos de dias atípicos, para que houvesse uma melhor representação da ingestão habitual. Em seguida, as quantidades descritas em medidas caseiras foram convertidas em gramas e mililitros e, por fim, o software nutricional Nutrilife $e^{\circledast}$ foi utilizado para calcular os valores de quilocalorias totais e a distribuição diária dos macronutrientes (carboidratos, lipídios e proteínas).

\section{f. Critérios de inclusão e não inclusão}

Utilizaram-se prontuários de pacientes adultos com SM que apresentavam o campo "horas de sono" e o recordatório alimentar devidamente preenchidos na anamnese. Prontuários com tais dados incompletos não foram incluídos neste estudo, de acordo com o observado no fluxograma abaixo. Outros critérios estão descritos em artigo de Araújo et al. (2019).

Figura 1 - Fluxograma do estudo

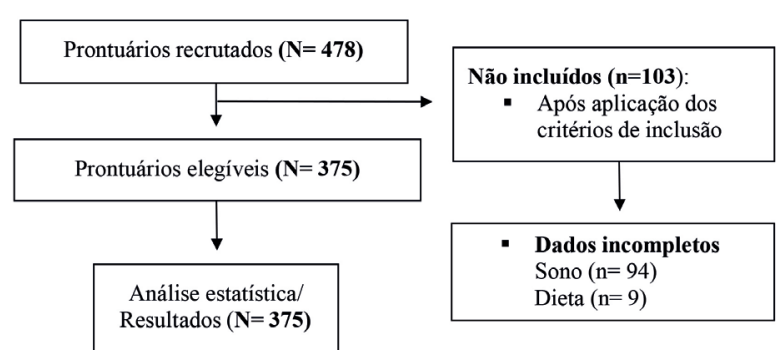

Fonte: Dados da pesquisa. 
Os dados dos pacientes foram tabulados em planiIhas do Microsoft Excel versão 2013 e analisados com o auxílio do software Statistical Package for the Social Sciences (SPSS) v. 20.0 para Windows. Estatísticas descritivas para dados sociodemográficos e cofatores da SM foram apresentadas em frequência absoluta e relativa ou média e desvio padrão, de acordo com as características das variáveis. As variáveis foram ajustadas de acordo com idade e sexo e as diferenças de quilocalorias e macronutrientes entre os grupos foram avaliadas através do teste Qui-Quadrado. Consideraram-se significativos valores de $p<0,05$.

\section{RESULTADOS}

A Tabela 1 sumariza as informações sociodemográficas da população do estudo, constituída por 375 indivíduos adultos de ambos os sexos. Destaca-se o grande número de participantes do sexo feminino e com idade a partir de 40 anos.

Tabela 1 - Dados sociodemográficos da população do estudo

\begin{tabular}{|c|c|c|}
\hline & $\mathbf{N}$ & $\%$ \\
\hline \multicolumn{3}{|l|}{ Sexo } \\
\hline Masculino & 59 & 15,7 \\
\hline Feminino & 316 & 84,3 \\
\hline \multicolumn{3}{|l|}{ Faixa etária } \\
\hline 20-39 anos & 41 & 10,9 \\
\hline $40-59$ anos & 334 & 89,1 \\
\hline \multicolumn{3}{|l|}{ Nível de Exercício Físico } \\
\hline Sedentário & 232 & 61,9 \\
\hline Exercício leve & 101 & 26,9 \\
\hline Exercício moderado & 41 & 10,9 \\
\hline Exercício intenso & 1 & 0,3 \\
\hline \multicolumn{3}{|l|}{ Escolaridade* } \\
\hline Analfabeto & 11 & 2,9 \\
\hline Fundamental incompleto-Fundamental completo & 141 & 37,7 \\
\hline Médio incompleto - Médio completo & 181 & 48,3 \\
\hline Superior incompleto - Superior completo & 41 & 11,0 \\
\hline \multicolumn{3}{|l|}{ Renda** } \\
\hline Até 1 Salário Mínimo & 136 & 36,6 \\
\hline De 1 a 3 Salários Mínimos & 186 & 50,0 \\
\hline De 3 a 5 Salários Mínimos & 43 & 11,6 \\
\hline Mais de 5 Salários Mínimos & 7 & 1,9 \\
\hline \multicolumn{3}{|l|}{ Ocupação*** } \\
\hline Desempregado & 65 & 17,5 \\
\hline Ativo & 163 & 43,9 \\
\hline Atividade do Lar & 85 & 22,9 \\
\hline Aposentado/ Licença & 58 & 15,7 \\
\hline
\end{tabular}

Fonte: Dados da pesquisa.

Legenda: ${ }^{*} 1, * * 3 e^{* * *} 4$ participantes não tinham essa informação; \%: frequência; salário mínimo atual no valor de $R \$ 1.100,00$.

Quanto aos cofatores da SM, com exceção da circunferência da cintura elevada, considerada pela IDF um critério obrigatório para a sua definição, a pressão arterial elevada ou diagnóstico de HAS foi o parâmetro de maior frequência. Em seguida, observaram-se níveis reduzidos de colesterol HDL, glicemia de jejum alterada ou diagnóstico de $\mathrm{DM}$ e, por último, hipertrigliceridemia. Esses dados são apresentados na Tabela 2.

Tabela 2 - Frequência dos cofatores da SM na população do estudo

\begin{tabular}{lrr}
\hline Pressão Arterial alterada ou HAS* & $n$ & $\%$ \\
$\quad$ Não & 21 & 5,6 \\
Sim & 353 & 94,4 \\
Glicemia de jejum alterada ou DM & & \\
$\quad$ Não & 93 & 24,8 \\
$\quad$ Sim & 282 & 75,2 \\
HDL-colesterol & 59 & 15,7 \\
$\quad$ Adequado & 316 & 84,3 \\
$\quad$ Alterado & & \\
Triglicerídeos & 109 & 29,1 \\
$\quad$ Adequado & 266 & 70,9 \\
$\quad$ Alterado & & \\
\hline
\end{tabular}

Fonte: Dados da pesquisa.

Legenda: HAS: Hipertensão Arterial Sistêmica; DM: Diabetes Mellitus. *1 participante não tinha informação sobre este parâmetro, porém tinham, ao menos, 3 outros cofatores da SM.

\%: frequência

A Tabela 3 mostra a frequência de horas de sono dos participantes. Observou-se que a maioria dos indivíduos relatou uma curta duração de sono, sendo que a menor frequência encontrada foi de longa duração do sono.

Tabela 3 - Frequência da duração do sono em pacientes com SM

\begin{tabular}{lrr}
\hline Tempo de sono* & \multicolumn{1}{c}{ n } & \multicolumn{1}{l}{$\%$} \\
$\leq 6$ horas & 184 & 49,1 \\
$7-8$ Horas & 155 & 41,3 \\
$\geq 9$ horas & 36 & 9,6 \\
Total & 375 & 100 \\
\hline
\end{tabular}

Fonte: Dados da pesquisa.

Legenda: *Curta duração: $\leq 6$ horas/noite; adequada: 7-8 horas/noite; longa duração: $\geq 9 /$ noite

\%: frequência

Com relação aos aspectos dietéticos, a média de consumo energético foi de 1679,6 quilocalorias, 222,6 gramas de carboidratos, 82,3 gramas de proteína e 49,6 gramas de lipídios. Houve uma discreta variação de quilocalorias, carboidratos e lipídios entre os grupos de curta e longa duração do sono, correspondente a 110 quilocalorias, 18 gramas e 7 gramas, respectivamente. A variação de proteína foi ainda menor, representando apenas 2 gramas, porém com maior ingestão proteica no grupo de longa duração do sono. Não foi encontrada associação estatisticamente significativa entre a duração do sono e o consumo energético diário total de carboidratos, proteínas e lipídeos, conforme apresentado na Tabela 4. 
Tabela 4 - Horas de sono, distribuição calórica e de macronutrientes em indivíduos com SM

\begin{tabular}{lrrrc}
\hline & \multicolumn{1}{c}{$\boldsymbol{5}$ h h* } & $\mathbf{7 - 8} \mathbf{h}^{*}$ & $\mathbf{2 9} \mathbf{h}^{*}$ & p valor** \\
Energia(Kcal) & $1721(879)$ & $1707(931)$ & $1611(596)$ & 0,957 \\
CHO (g) & $232(120)$ & $222(99)$ & $214(73)$ & 0,975 \\
PTN (g) & $82(45)$ & $81(43)$ & $84(41)$ & 0,865 \\
LIP (g) & $53(36)$ & $50(35)$ & $46(28)$ & 0,382 \\
\hline
\end{tabular}

Fonte: Dados da pesquisa.

Legenda: $h$ : horas; Kcal: quilocalorias; CHO: carboidratos; PTN: proteínas; LIP: lipídios; g: gramas; *Média (desvio-padrão); **Teste Qui-quadrado

\section{DISCUSSÃO}

A ideia de que o sono influencia o consumo alimentar tem sido amplamente discutida nos últimos anos, com evidências de que tanto a curta, quanto a longa duração do sono, podem desencadear comportamentos alimentares desfavoráveis (DASHIT et al., 2015). Essa relação parece ser maior no contexto do deficit de sono, em decorrência de fatores como o desequilíbrio nos hormônios grelina e leptina, o qual induz alterações no apetite (FRANK et al., 2017; LUNDAHL; NELSON, 2015), maior oportunidade para se alimentar (PATTERSON et al., 2014) e maior processamento de estímulos hedônicos no cérebro, que geram um mecanismo de busca por recompensa (CHAPUT, 2014; CASTRO et al., 2019; ST-ONGE et al., 2014).

Contudo, em nossa investigação não foi encontrada associação estatisticamente significativa entre a duração do sono e o consumo energético diário total, o que também foi visto por Nakayama et al. (2021) e Castro et al. (2019). Todavia, um menor valor médio de quilocalorias totais foi observado no grupo de longa duração do sono, corroborando a hipótese de que a longa duração do sono leva a um menor consumo de energia nas refeições principais - desjejum, almoço e jantar (KANT; GRAUBARD, 2014; KIM; DEROO; SANDLER, 2011).

Em oposição aos nossos achados, estudos sugerem que os distúrbios de sono, em especial nas condições de privação, promovem maior ingestão calórica (AL KHATIB et al., 2017; BAYON et al., 2014; DASHTI et al., 2015; GALLI et al., 2013). Embora haja um acréscimo modesto no gasto energético, como resultado de horas prolongadas de vigília, isso parece ser muitas vezes substituído por aumentos consideráveis na ingestão alimentar (ALIMENTI et al., 2021; DASHTI et al., 2015).

Quanto à composição da dieta, nossos resultados também não encontraram associação entre o tempo de sono e o consumo de carboidratos, proteínas e lipídios. A ausência de associação entre o sono e os macronutrientes também foi descrita por Dashti et al. (2015) e Alimenti et al. (2021).

Em contraste, pesquisadores relataram que lipídios e açúcares têm sido destacados como nutrientes de maior escolha durante os períodos de restrição do sono (NISHIURA; NOGUCHI; HASHIMOTO, 2010; ST-ONGE et al., 2011). Em tese, a preferência por esses alimentos de alta densidade energética implicaria uma maior ingestão calórica
(NISHIURA; NOGUCHI; HASHIMOTO, 2010). Entretanto, Castro et al. (2019) notaram que a duração do sono afetou mais a programação das refeições e a distribuição de energia e de macronutrientes do que a ingestão calórica total durante o ciclo de 24 horas. Em outra via, porém em menor grau, há evidências de que a longa duração do sono pode levar a períodos inadequados de refeições, maior ingestão de doces e gorduras (KANT; GRAUBARD, 2014; KIM; DEROO; SANDLER, 2011).

As horas de sono têm diminuído continuamente nas últimas décadas (DASHTI et al., 2015; REITER et al., 2012), como resultado de um fenômeno conhecido como privação voluntária de sono, em função das mudanças na dinâmica de trabalho e no estilo de vida das pessoas (CAPPUCCIO et al., 2010). Por sua vez, postula-se que esse novo padrão tenha relação com a SM, cuja prevalência também está em ascensão (JU; CHOI, 2013; KIM et al., 2018). Iftikhar et al. (2015) concluíram que as pessoas que relatam uma duração do sono inferior a 5 horas têm uma chance 1,5 vezes maior de desenvolver a SM.

Seguindo essa perspectiva, em nosso estudo, quase metade dos participantes relatou sono curto ( $\leq 6$ horas por noite). Lima, Francisco e Barros (2012) e Castro et al. (2019), que também avaliaram adultos brasileiros, notaram uma maior prevalência de sono adequado; porém as populações avaliadas não eram compostas exclusivamente de pacientes com SM, como a nossa. Ainda, fatores sociodemográficos também podem estar por trás dessas variações.

Nossa amostra foi caracterizada em sua maioria por mulheres e em ocupação ativa no mercado de trabalho, características que podem levar a uma redução das horas de sono. Além das variações hormonais e fisiológicas que podem variar a arquitetura do sono durante o ciclo menstrual, gravidez e menopausa, distúrbios de sono podem estar mais presentes na população feminina devido à sobrecarga de trabalho (mãe, dona de casa e profissional), fazendo com que os momentos de descanso sejam voltados para realização de diversas tarefas (GAJARDO et al., 2021; MOREIRA et al., 2013).

Sobre o trabalho, fatores psicossociais como alta demanda, estresse, assédio moral e desequilíbrio no binômio esforço-recompensa podem desencadear problemas com o sono (GAJARDO et al., 2021).

O sedentarismo também está ligado ao sono inadequado. Em nossa investigação foi observado que um número expressivo de pessoas $(61,9 \%)$ não realizava exercício físico, cujo efeito na melhora do sono está relacionado às hipóteses de termorregulação, conservação de energia e restauração corporal. Indivíduos que se exercitam regularmente sentem-se menos cansados durante o dia, adormecem mais rápido e passam mais tempo nos estágios de sono profundo, indicando que o exercício físico pode ser uma intervenção positiva no sono (MOREIRA et al., 2013). Moreira et al. (2013) demonstraram que pessoas fisicamente ativas tinham um padrão de sono melhor do que as sedentárias. 
Gajardo et al. (2021), ao avaliar os fatores associados aos distúrbios de sono na população brasileira, também verificaram que a ocorrência de problemas com o sono foi mais frequente entre as mulheres, com o aumento da idade e com comportamento sedentário.

Existem evidências de que a duração do sono também pode estar associada à idade, ao nível de escolaridade e à renda. Em nossa população, houve um maior número de indivíduos com idade superior a 40 anos. Tal qual Gajardo et al. (2021), Lima, Francisco e Barros (2012) encontraram maior prevalência de sono curto com o aumento da idade, indicando que há mais distúrbios de sono à medida em que a idade vai aumentando. Em relação à renda, foi observado que a maioria dos participantes ganhava até três salários mínimos mensais, o que suporta a teoria de que há uma maior prevalência de sono adequado entre os estratos socioeconômicos mais elevados (LIMA; FRANCISCO; BARROS, 2012; WHINNERY et al., 2014).

Sobre a escolaridade, há resultados divergentes. Enquanto Whinnery et al.(2014) observaram menor duração do sono nos níveis de educação mais baixos, quando comparados a universitários, no estudo de Lima, Francisco e Barros (2012), o sono curto foi mais prevalente entre os que tinham nível superior. Em nossa amostra, havia mais pessoas com escolaridade até o ensino médio completo. É possível que os demais fatores sociodemográficos tenham causado maior influência na duração do sono. É importante ressaltar que a saúde do sono é um conceito multidimensional e sua avaliação deve incluir indicadores quantitativos (duração, latência e número de despertares) e qualitativos, como profundidade do sono e satisfação geral (LIAN et al.,2019).

Este estudo apresenta algumas limitações. Primeiramente, tanto a duração do sono, quanto a dieta, foram estimadas apenas por meio de informações autorrelatadas, ainda que essas ferramentas sejam um método mais prático e econômico para uso em pesquisas (CASTRO et al., 2019). Em segundo lugar, os dados de sono não contemplaram informações sobre a realização de cochilos diurnos, que podem interferir nas horas dormidas no período noturno. Aspectos qualitativos sobre o sono e a dieta também não foram investigados. Por fim, a ausência de um grupo sem SM para comparar os resultados não permitiu uma análise mais ampla sobre a relação entre sono e consumo alimentar.

\section{CONCLUSÃO}

No presente estudo, foi observado que quase metade dos participantes relatou uma curta duração de sono. Fatores sociodemográficos, incluindo gênero, renda, ocupação e nível de exercício físico podem justificar este resultado. Contudo, não foram encontradas associações significativas entre a duração do sono e o consumo energético e de macronutrientes em uma amostra de pacientes com SM. Sendo o comportamento alimentar um importante fator na etiologia da SM, pesquisas fu- turas são necessárias para compreender o impacto do sono na dieta.

\section{REFERÊNCIAS}

ALIMENTI, K. et al. Habitual sleep duration, daytime napping, and dietary intake: A mendelian randomization study. Current Developments in Nutrition, [s.l.], v. 5, n. 3, p. nzab019, 2021.

AL KHATIB, H. K. et al. The effects of partial sleep deprivation on energy balance: a systematic review and meta-analysis. European Journal of Clinical Nutrition, London, v. 71, n. 5, p. 614-624, 2017.

ALBERTI, K. G. M. M.; ZIMMET, P.; SHAW, J. Metabolic syndrome-a new world-wide definition. A consensus statement from the international diabetes federation. Diabetic Medicine, Chichester, v. 23, n. 5, p. 469480, 2006.

ARAUJO, E. M. et al. Genetic and oral tests for the diagnosis of lactose intolerance in mixed-ancestry Brazilians with metabolic syndrome. Lifestyle Genomics, [s.I], v. 12, n. 1-6, p. 1-9, 2019.

BAYON, V. et al. Sleep debt and obesity. Annals of Medicine, Leawood, v. 46, n. 5, p. $264-272,2014$.

CAPPUCCIO, F. P. et al. Quantity and quality of sleep and incidence of type 2 diabetes: a systematic review and meta-analysis. Diabetes Care, Alexandria, v. 33, n. 2, p. 414-420, 2010a.

CAPPUCCIO, F. P. et al. Sleep duration and all-cause mortality: a systematic review and meta-analysis of prospective studies. Sleep, New York, v. 33, n. 5, p. 585-592, 2010b.

CAPPUCCIO, F. P. et al. Sleep duration predicts cardiovascular outcomes: a systematic review and meta-analysis of prospective studies. European Heart Journal, London, v. 32, n. 12, p. 1484-1492, 2011.

CARVALHO, M. H. C. de. I Diretriz brasileira de diagnóstico e tratamento da síndrome metabólica. Arquivos Brasileiros de Cardiologia, São Paulo, v. 84, p. 1-28, 2005.

CASTRO, M. A. de et al. Eating behaviours and dietary intake associations with self-reported sleep duration of free-living Brazilian adults. Appetite, London, v. 137, p. 207-217, 2019.

CHAPUT, J-P. Sleep patterns, diet quality and energy balance. Physiology \& Behavior, Elmsford, v. 134, p. 86-91, 2014.

DASHTI, H. S. et al. Short sleep duration and dietary intake: epidemiologic evidence, mechanisms, and health implications. Advances in Nutrition, [s.l.], v. 6, n. 6, p. 648-659, 2015.

FRANK, S. et al. Diet and sleep physiology: public health and clinical implications. Frontiers in Neurology, [s.I.], v. 8, p. 393, 2017.

GAJARDO, Y. Z. et al. Problemas com o sono e fatores associados na população brasileira: Pesquisa Nacional de Saúde, 2013. Ciência \& Saúde Coletiva, Rio de Janeiro, v. 26, p. 601-610, 2021.

GALLI, G. et al. Inverse relationship of food and alcohol intake to sleep measures in obesity. Nutrition \& Diabetes, [s.l.], v. 3, n. 1, p. e58-e58, 2013.

GRANDNER, M. A. et al. Dietary nutrients associated with short and long sleep duration. Data from a nationally representative sample. Appetite, London, v. 64, p. $71-80,2013$.

IFTIKHAR, I. H. et al. Sleep duration and metabolic syndrome. An updated dose-risk metaanalysis. Annals of the American Thoracic Society, v. 12, n. 9, p. 1364-1372, 2015.

JU, S. Y.; CHOI, W. S. Sleep duration and metabolic syndrome in adult 
populations: a meta-analysis of observational studies. Nutrition \& Diabetes, [s.l.], v. 3, n. 5, p. e65-e65, 2013.

KANT, A. K.; GRAUBARD, B. I. Association of self-reported sleep duration with eating behaviors of American adults: NHANES 2005-2010. The American Journal of Clinical Nutrition, [s.I.], v. 100, n. 3, p. 938-947, 2014.

$\mathrm{KIM}, \mathrm{C}$. E. et al. Association between sleep duration and metabolic syndrome: a cross-sectional study. BMC Public Health, London, v. 18, n. 1 , p. 1-8, 2018

KIM, S.; DEROO, L. A.; SANDLER, D. P. Eating patterns and nutritional characteristics associated with sleep duration. Public Health Nutrition, Wallingford, v. 14, n. 5, p. 889-895, 2011.

LIAN, Y. et al. Association between sleep quality and metabolic syndrome: A systematic review and meta-analysis. Psychiatry Research, Amsterdam, v. 274, p. 66-74, 2019.

LIMA, M. G.; FRANCISCO, P. M. S. B.; BARROS, M. B. de A. Sleep duration pattern and chronic diseases in Brazilian adults (ISACAMP, 2008/09). Sleep Medicine, Amsterdam, v. 13, n. 2, p. 139-144, 2012.

LUNDAHL, A.; NELSON, T. D. Sleep and food intake: a multisystem review of mechanisms in children and adults. Journal of Health Psychology, London, v. 20, n. 6, p. 794-805, 2015.

MOREIRA, L. P. et al. Comparação da qualidade do sono entre homens e mulheres ativos fisicamente. Revista Eletrônica Saúde e Ciência, [s.I], v. 3, n. 2, p. $38-49,2013$.

NAKAYAMA, H. et al. Distinct relevance of nightly sleep duration to metabolic, anthropometric, and lifestyle factors in patients with type 2 diabetes. Internal Medicine, Tokyo, v. 60, n. 5 p. 681-688, 2021.

NISHIURA, C.; NOGUCHI, J.; HASHIMOTO, H. Dietary patterns only partially explain the effect of short sleep duration on the incidence of obesity. Sleep, New York, v. 33, n. 6, p. 753-757, 2010.

PATTERSON, R. E. et al. Short sleep duration is associated with higher energy intake and expenditure among African-American and non-
Hispanic white

adults. The Journal of Nutrition, [s.I.], v. 144, n. 4, p. 461-466, 2014.

REITER, R. J. et al. Obesity and metabolic syndrome: association with chronodisruption, sleep deprivation, and melatonin suppression. Annals of Medicine, Helsinki, v. 44, n. 6, p. 564-577, 2012.

SANTOS, P. R.; FERRARI, G. S. L.; FERRARI, C. K. B. Diet, sleep and metabolic syndrome among a legal Amazon population, Brazil. Clinical Nutrition Research, [s.I.], v. 4, n. 1, p. 41-45, 2015.

ST-ONGE, M-P. et al. Short sleep duration increases energy intakes but does not change energy expenditure in normal-weight individuals. The American Journal of Clinical Nutrition, [s.I.], v. 94, n. 2, p. 410-416, 2011.

ST-ONGE, M-P.; MIKIC, A.; PIETROLUNGO, C. E. Effects of diet on sleep quality. Advances in Nutrition, Bethesda, v. 7, n. 5, p. 938-949, 2016.

ST-ONGE, M. P. et al. Sleep restriction increases the neuronal response to unhealthy food in normal-weight individuals. International Journal of Obesity, London, v. 38, n. 3, p. 411-416, 2014.

WHINNERY, J. et al. Short and long sleep duration associated with race/ ethnicity, sociodemographics, and socioeconomic position. Sleep, London, v. 37, n. 3, p. 601-611, 2014.

WU, Y.; ZHAI, L.; ZHANG, D. Sleep duration and obesity among adults: a meta-analysis of prospective studies. Sleep Medicine, Amsterdam, $\mathrm{v}$. 15, n. 12, p. $1456-1462,2014$.

YIN, J. et al. Relationship of sleep duration with all-cause mortality and cardiovascular events: a systematic review and dose-response metaanalysis of prospective cohort studies. Journal of the American Heart Association, [s.l.], v. 6, n. 9, p. e005947, 2017.

ZURAIKAT, F. M. et al. Measures of poor sleep quality are associated with higher energy intake and poor diet quality in a diverse sample of women from the go red for women strategically focused research network. Journal of the American Heart Association, [s.l.], v. 9, n. 4, p. e014587, 2020.

Submetido em: $19 / 11 / 2021$

Aceito em: 20/11/2021 